\title{
IN VITRO SCHISTOSOMICIDAL ACTIVITY OF TRITERPENOIDS FROM THE AFRICAN PLANT MOMORDICA BALSAMINA
}

\author{
Ramalhete $C^{1}$, Magalhães $L^{2,3}$, Rodrigues $V^{3}$, Mulhovo $S^{4}$, Da Silva Filho AA ${ }^{5}$, Ferreira MJU'
}

${ }^{1}$ Research Institute for Medicines and Pharmaceutical Sciences (iMed.UL), Faculdade de Farmácia, Universidade de Lisboa, Av. das Forças

Armadas, 1600-083, Lisboa, Portugal; ${ }^{2}$ Núcleo de Ciências Exatas e Tecnológicas, Universidade de Franca, São Paulo, Brazil; ${ }^{3}$ Faculdade de

Medicina de Ribeirão Preto, Universidade de São Paulo, Ribeirão Preto, Brazil; ${ }^{4}$ Departamento de Ciências Agro-Pecuárias, Universidade

Pedagógica, Moçambique; 5 Departamento de Ciências Farmacêuticas, Faculdade de Farmácia, Universidade Federal de Juiz de Fora, 36036-330,

Juiz de Fora, MG, Brazil. mjuferreira@ff.ul.pt

\section{INTRODUCTION}

Schistosomiasis, also known as bilharzia, is a chronic liver and intestinal parasitic disease caused by trematode worms of the genus Schistosoma. Among the five major species of human schistosomes, Schistosoma mansoni is the most prevalent, being endemic in 54 countries. Praziquantel is the only available drug against all forms of schistosomiasis. The development of praziquantel resistance is a great concern and new drugs are urgently needed [1].

Momordica balsamina L. (Cucurbitaceae), commonly known as African pumpkin, is a vegetable widespread in tropical and subtropical regions that has been used as food, mainly in sub-Saharan Africa. It has also been widely used in traditional medicine in Africa to treat various disease symptoms, mostly diabetes and malaria.

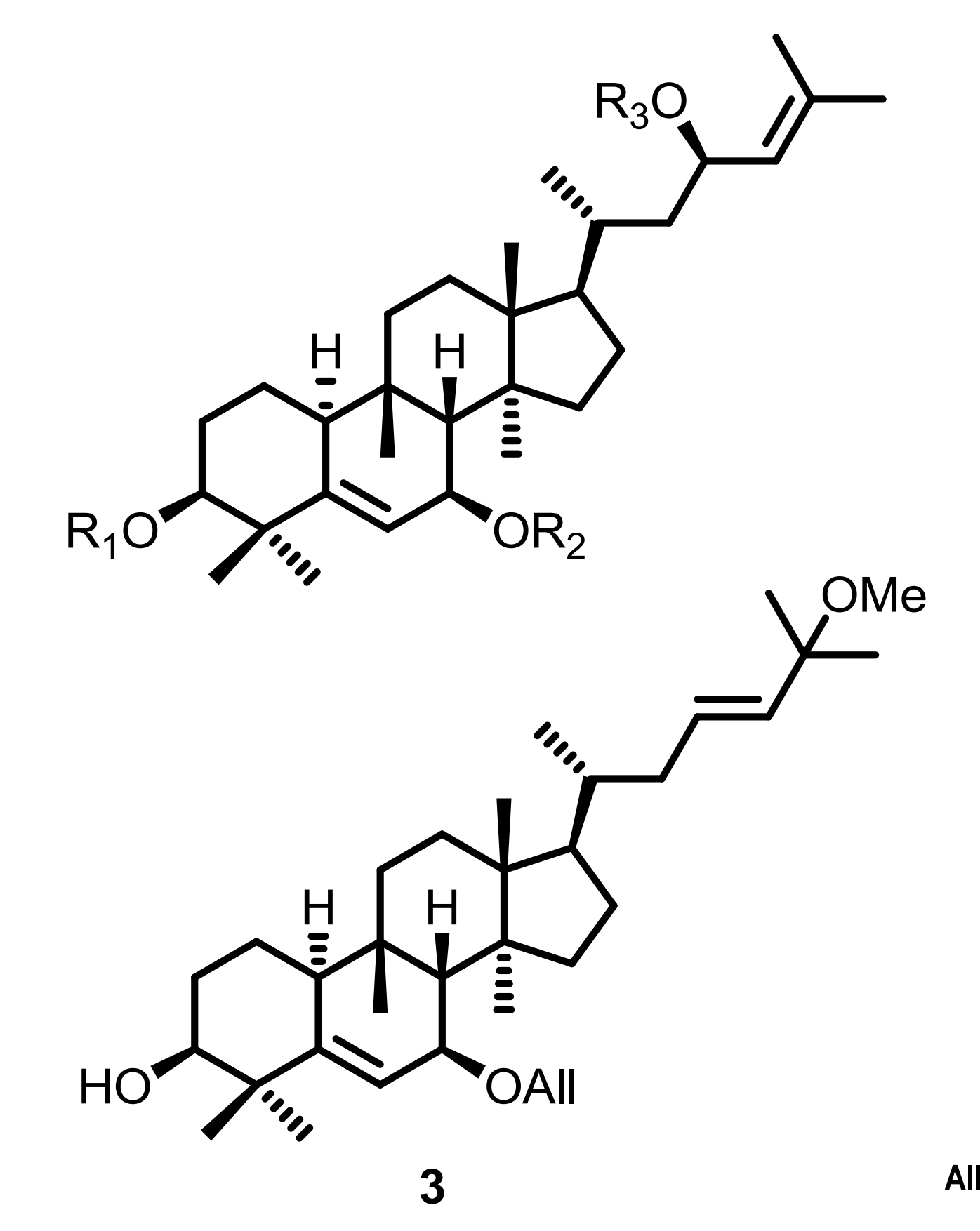

$1: \mathrm{R}_{1}=\mathrm{H} ; \mathrm{R}_{2}=\mathrm{CH}_{3} ; \mathrm{R}_{3}=\mathrm{H}$

$2: R_{1}=H ; R_{2}=H ; R_{3}=H$

$4: R_{1}=H ; R_{2}=A l l ; R_{3}=H$

$5: R_{1}=H ; R_{2}=G l u ; R_{3}=H$

$6: \mathrm{R}_{1}=\mathrm{H} ; \mathrm{R}_{2}=\mathrm{CH}_{3} ; \mathrm{R}_{3}=\mathrm{COCH}_{3}$

$7: \mathrm{R}_{1}=\mathrm{COCH}_{3} ; \mathrm{R}_{2}=\mathrm{CH}_{3} ; \mathrm{R}_{3}=\mathrm{COCH}_{3}$

$8: \mathrm{R}_{1}=\mathrm{H} ; \mathrm{R}_{2}=\mathrm{CH}_{3} ; \mathrm{R}_{3}=\mathrm{COCH}_{2} \mathrm{CH}_{3}$

$9: \mathrm{R}_{1}=\mathrm{H} ; \mathrm{R}_{2}=\mathrm{CH}_{3} ; \mathrm{R}_{3}=p$-methoxyBz

$10: \mathrm{R}_{1}=p$-methoxyBz; $\mathrm{R}_{2}=\mathrm{CH}_{3} ; \mathrm{R}_{3}=p$-methoxyBz

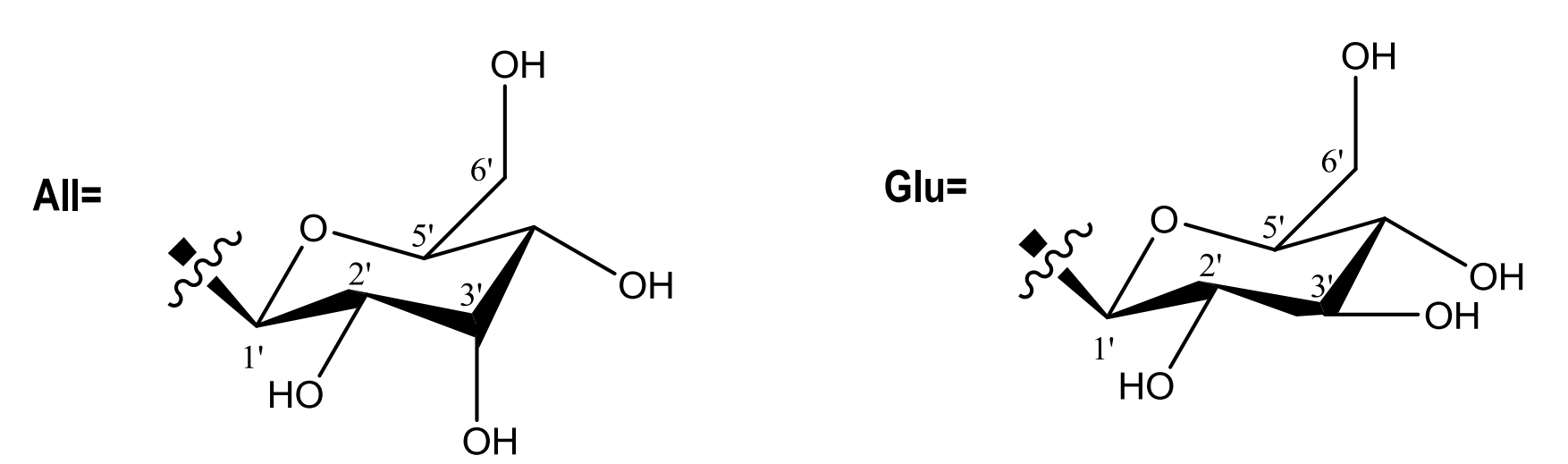

Figure 3. Chemical structures of the isolated compounds (1 $-\mathbf{5})$

\section{RESULTS AND DISCUSSION}

For the in vitro test with S. mansoni, compounds 1-10 were dissolved in 10\% DMSO and used in concentrations ranging between 5 to $100 \mu \mathrm{M}$, which were added to the medium containing one adult pair. Parasites were evaluated for their general condition: motor activity, alterations in the tegument, mortality rate, and egg production and development.

A remarkable schistosomicidal activity was observed for karavilagenin $\mathbf{C}$ and balsaminol $\mathbf{F}$ at 50 and $100 \mu \mathrm{M}$, which caused the death of all S. mansoni adult worms after $24 \mathrm{~h}$ of incubation. Both compounds, at 10-50 $\mathrm{MM}$, induced significant reductions in the motor activity of the worms (data no shown) and significantly decreased the egg production (Fig. 3). Furthermore, they were able (at 10-100 $\mu \mathrm{M}$ ) to separate the adult worm pairs into male and female after $24 \mathrm{~h}$ (data no shown).

\section{CONCLUSION}

Our results indicate that karavilagenin $\mathbf{C}$ and balsaminol F possesses in vitro schistosomicidal activity against S. Mansoni adult worms. Then, cucurbitane derivatives might be used, in the future, as lead compounds for the development of new schistosomicidal agents.

\section{REFERENCES}

1. WHO (2010). Fact sheet no 115; 2. Ramalhete, C. et al. (2010) Bioorg. Med. Chem. 18, 5254-60.; 3. Ramalhete, C. et al. (2011) Bioorg. Med. Chem. 19, 330-8; 4. Magalhães L.G. et al. (2010) Parasitol Res 106: 395-401; 5. Magalhães L.G. et al. (2009) Parasitol Res 104: $1197-120$.
Figure 1. Momordica balsamina.
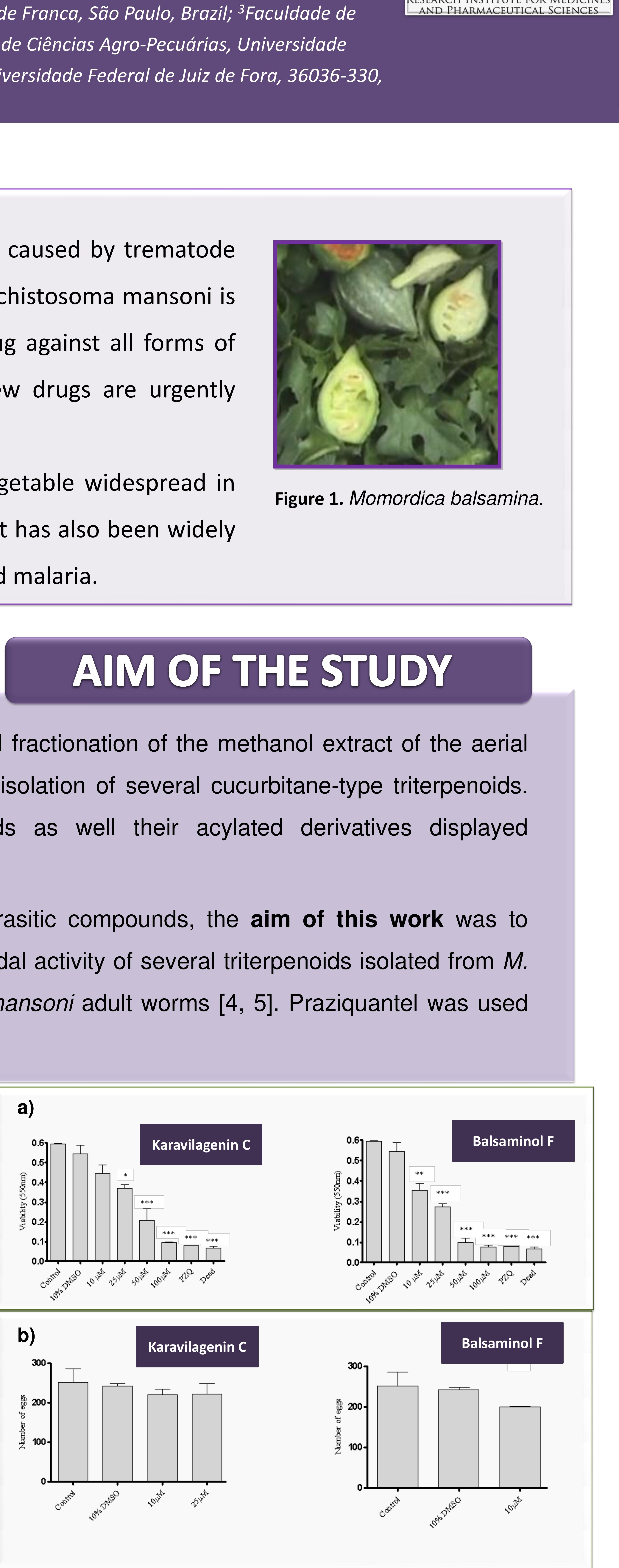

Figure 3. a) In vitro effects of active curcubitane-type triterpenoids (1, 2) on the viability of the $S$. mansoni adult worms. b) In vitro effects of curcubitane-type triterpenoids $\mathbf{1}, 2$ in the egg production. ${ }^{*} P<0.05$, ${ }^{* *}$ $P<0.01$ and ${ }^{* \star} P<0.001$. 